\title{
Asymptotic Normality in Monte Carlo Integration
}

\author{
By Masashi Okamoto
}

\begin{abstract}
To estimate a multiple integral of a function over the unit cube, Haber proposed two Monte Carlo estimators $J_{1}^{\prime}$ and $J_{2}^{\prime}$ based on $2 N$ and $4 N$ observations, respectively, of the function. He also considered estimators $D_{1}^{2}$ and $D_{2}^{2}$ of the variances of $J_{1}^{\prime}$ and $J_{2}^{\prime}$, respectively. This paper shows that all these estimators are asymptotically normally distributed as $N$ tends to infinity.
\end{abstract}

1. Introduction. Monte Carlo integration is a method to estimate the value of a definite integral of a given real-valued function over a finite region (say, a cube) by observing the value of the function only at a finite number of points in the region which are chosen suitably and stochastically. Kitagawa [4] proposed several estimating methods but he was concerned mainly with the case when the function has a certain prior distribution.

Haber [1] , [2] proposed a mesh estimator of the integral and then improved it by means of the idea of "antithetic variates" due to Hammersley and Morton [3]. Specifically, let $f$ be a real-valued function defined over the unit cube $G_{s}$ in the $s$ space and set $I=\int_{G_{s}} f$. Let $A_{r}(r=1, \ldots, N)$ be a family of congruent subcubes arising by partitioning $G_{s}$ so that the interval $[0,1]$ on the $x^{i}$-axis is divided into $n_{i}$ equal subintervals for each $i=1, \ldots, s$, where $N=n_{1} \cdots n_{s}$. Let $x_{r}$ be a random point in $A_{r}$ chosen independently for each $r$ and let $x_{r}^{\prime}=2 c_{r}-x_{r}$, where $c_{r}$ stands for the center of $A_{r}$. Then

$$
J_{1}=\frac{1}{N} \sum_{r=1}^{N} f\left(x_{r}\right)
$$

and

$$
J_{2}=\frac{1}{N} \sum_{r=1}^{N} \frac{f\left(x_{r}\right)+f\left(x_{r}^{\prime}\right)}{2}
$$

are unbiased estimators of $I$ based on $N$ and $2 N$ observations of $f$, respectively.

To estimate the variances of $J_{1}$ and $J_{2}$ we need replications of observations. Let $z_{r}$ be another random point in $A_{r}$, the $z_{r}$ being chosen independently of each other and also of the $x$ 's. Define similarly $z_{r}^{\prime}=2 c_{r}-z_{r}$. Then

and

$$
J_{1}^{\prime}=\frac{1}{N} \sum_{r=1}^{N} \frac{f\left(x_{r}\right)+f\left(z_{r}\right)}{2}
$$

$$
J_{2}^{\prime}=\frac{1}{N} \sum_{r=1}^{N} \frac{f\left(x_{r}\right)+f\left(x_{r}^{\prime}\right)+f\left(z_{r}\right)+f\left(z_{r}^{\prime}\right)}{4}
$$

Received August 26, 1975 ; revised January 27, 1976.

AMS (MOS) subject classifications (1970). Primary 65D30; Secondary 62E20, 60F05.

Key words and phrases. Monte Carlo integration, Haber's estimators, asymptotic normality. Lyapunov condition. 
are again unbiased estimators of $I$ based on $2 N$ and $4 N$ observations, respectively. By using these random points, the variances of $J_{1}^{\prime}$ and $J_{2}^{\prime}$ can be estimated unbiasedly by

$$
D_{1}^{2}=\frac{1}{4 N^{2}} \sum_{r=1}^{N}\left\{f\left(x_{r}\right)-f\left(z_{r}\right)\right\}^{2}
$$

and

$$
p_{2}^{2}=\frac{1}{4 N^{2}} \sum_{r=1}^{N}\left\{\frac{f\left(x_{r}\right)+f\left(x_{r}^{\prime}\right)}{2}-\frac{f\left(z_{r}\right)+f\left(z_{r}^{\prime}\right)}{2}\right\}^{2},
$$

respectively.

For $k=1$ and 2 let $C^{k}$ denote the set of all real-valued functions defined over $G_{s}$ and having continuous $k$ th order partial derivatives. In the sequel we say just " $N \rightarrow \infty$ " to indicate that $n_{i} \rightarrow \infty$ for every $i=1, \ldots, s$. Put $n=\min \left(n_{1}, \ldots, n_{s}\right)$. Haber proved that if $f \in C^{1}$, then

$$
\operatorname{var}\left(J_{1}\right)=\tau_{N}^{2}\left(J_{1}\right)+o\left(\left(N n^{2}\right)^{-1}\right) \quad \text { as } N \rightarrow \infty,
$$

and also that if $f \in C^{2}$, then

$$
\operatorname{var}\left(J_{2}\right)=\tau_{N}^{2}\left(J_{2}\right)+o\left(\left(N n^{4}\right)^{-1}\right) \quad \text { as } N \rightarrow \infty,
$$

where

$$
\begin{aligned}
\tau_{N}^{2}\left(J_{1}\right)= & \frac{1}{12 N} \sum_{i=1}^{s} \frac{1}{n_{i}^{2}} \int_{G_{s}}\left(\frac{\partial f}{\partial x^{i}}\right)^{2} \\
\tau_{N}^{2}\left(J_{2}\right)= & \frac{1}{1440 N}\left\{2 \sum_{i=1}^{s} \frac{1}{n_{i}^{4}} \int_{G_{s}}\left(\frac{\partial^{2} f}{\partial\left(x^{i}\right)^{2}}\right)^{2}\right. \\
& \left.+5 \sum_{i \neq j=1}^{s} \frac{1}{\left(n_{i} n_{j}\right)^{2}} \int_{G_{s}}\left(\frac{\partial^{2} f}{\partial x^{i} \partial x^{j}}\right)^{2}\right\}
\end{aligned}
$$

Furthermore, since $J_{k}^{\prime}(k=1,2)$ is the average of two independent realizations of $J_{k}$, its variance is half as large as that of $J_{k}$. In estimating $I$ by $J_{k}^{\prime}$, Haber used $D_{k}$ as a measure of the error, assuming that $J_{k}^{\prime}$ is approximately normally distributed.

The purpose of this paper is to show, first, that $J_{k}(k=1,2)$ is asymptotically normally distributed with mean $I$ and variance $\tau_{N}^{2}\left(J_{k}\right)$ as $N \rightarrow \infty$. This implies the asymptotic normality of $J_{k}^{\prime}$. Next, it is shown that $D_{k}^{2}(k=1,2)$ is asymptotically normally distributed with mean $\operatorname{var}\left(J_{k}^{\prime}\right)$ and a proper variance.

2. Asymptotic Normality of $J_{1}$ and $J_{2}$. Before stating the theorems we introduce some notations. Let

$$
\begin{aligned}
& I_{r}=E\left\{f\left(x_{r}\right)\right\}=N \int_{A_{r}} f \\
& \delta_{r}=\left(\delta_{r}^{1}, \ldots, \delta_{r}^{\mathcal{S}}\right)=x_{r}-c_{r}, \\
& f_{r}=f\left(c_{r}\right), \quad f_{r}^{i}=\frac{\partial f}{\partial x^{i}}\left(c_{r}\right) \text { and } f_{r}^{i j}=\frac{\partial^{2} f}{\partial x^{i} \partial x^{j}}\left(c_{r}\right),
\end{aligned}
$$


where $x=\left(x^{1}, \ldots, x^{s}\right)$, for $i, j=1, \ldots, s$, and $r=1, \ldots, N$. The $L_{2}$-norm wilı be denoted by \|\| .

THEOREM 1. Assume $f \in C^{1}$. As $N \rightarrow \infty, J_{1}$ is asymptotically normally distributed with mean I and variance $\tau_{N}^{2}\left(J_{1}\right)$ defined in (1.9).

Proof. Since $I=\Sigma_{r=1}^{N} I_{r} / N$, it holds that

$$
J_{1}-I=\frac{1}{N} \sum_{r=1}^{N}\left\{f\left(x_{r}\right)-I_{r}\right\} .
$$

A Taylor series expansion of $f\left(x_{r}\right)$ around the point $c_{r}$ gives

$$
f\left(x_{r}\right)=f_{r}+\sum_{i=1}^{s} \delta_{r}^{i} f_{r}^{i}+R_{1 r}
$$

where the remainder $R_{1 r}$ has the following property in view of the uniform continuity of $\partial f / \partial x^{i}$ in $G_{s}$ : for any $\epsilon>0,\left|R_{1 r}\right| \leqslant \epsilon\left\|\delta_{r}\right\|$ for every $r$, provided $N$ is sufficiently large.

Again, by a Taylor expansion we have

$$
I_{r}=f_{r}+R_{2 r}
$$

where $\left|R_{2 r}\right| \leqslant \epsilon / n$ for every $r$, provided $N$ is sufficiently large. Substitution of (2.2) and (2.3) into (2.1) yields

$$
J_{1}-I=S_{N}+R_{N}
$$

where

$$
S_{N}=\frac{1}{N} \sum_{r=1}^{N} \sum_{i=1}^{s} \delta_{r}^{i} f_{r}^{i}, \quad R_{N}=\frac{1}{N} \sum_{r=1}^{N}\left(R_{1 r}-R_{2 r}\right) .
$$

Since $E\left(J_{1}\right)=I$, to prove the theorem it remains only to verify the following three propositions:

(i) $\operatorname{var}\left(S_{N}\right) \sim \tau_{N}^{2}\left(J_{1}\right)$ as $N \rightarrow \infty$, where the symbol $\sim$ means that the ratio of the two sides tends to one,

(ii) the sequence $S_{N}$ satisfies the Lyapunov condition of the central limit theorem (see, e.g., Loève [5, p. 275]), and

(iii) $\operatorname{var}\left(R_{N}\right)=o\left(\left(N n^{2}\right)^{-1}\right)$ as $N \rightarrow \infty$.

In fact, in the right-hand side of the identity

$$
\frac{J_{1}-I}{\tau_{N}}=\frac{S_{N}-E\left(S_{N}\right)}{\tau_{N}}+\frac{R_{N}-E\left(R_{N}\right)}{\tau_{N}}
$$

the first term converges to the standardized normal distribution because of (i) and (ii), whereas the second term converges in probability to zero because of (iii) and the Chebyshev inequality.

Now, part (i) is essentially equivalent to Theorem 3 of Haber [1] or (1.7); but a proof is given here for the completeness of the proof. By definition, $\delta_{r}^{i}$ has a uniform distribution over the interval $\left[-1 /\left(2 n_{i}\right), 1 /\left(2 n_{i}\right)\right]$ independently of each other; and hence, $\operatorname{var}\left(\delta_{r}^{i}\right)=1 /\left(12 n_{i}^{2}\right)$, which implies that 


$$
\operatorname{var}\left(S_{N}\right)=\frac{1}{12 N^{2}} \sum_{r=1}^{N} \sum_{i=1}^{s} \frac{1}{n_{i}^{2}}\left(f_{r}^{i}\right)^{2} \sim \tau_{N}^{2}\left(J_{1}\right) \text { as } N \rightarrow \infty .
$$

$\operatorname{Re}\left(\right.$ ii). Define $X_{N r}=\Sigma_{i=1}^{s} \delta_{r}^{i} f_{r}^{i}$, then

$$
\sum_{r} E\left(X_{N r}^{2}\right)=N^{2} \operatorname{var}\left(S_{N}\right) \text {. }
$$

From the inequality $\left|\delta_{r}^{i}\right| \leqslant 1 /\left(2 n_{i}\right)$ it follows that

$$
\sum_{r} E\left|X_{N r}\right|^{3} \leqslant \frac{1}{8} \sum_{r}\left(\sum_{i}\left|f_{r}^{i}\right| / n_{i}\right)^{3} \sim \frac{N}{8} \int\left(\sum_{i} \frac{1}{n_{i}}\left|\frac{\partial f}{\partial x^{i}}\right|\right)^{3}=O\left(N n^{-3}\right) .
$$

By (i), (2.6) and (2.7)

$$
\left[\sum_{r} E\left|X_{N r}\right|^{3}\right] /\left[\sum_{r} E\left(X_{N r}^{2}\right)\right]^{3 / 2}=O\left(N^{-1 / 2}\right)=o(1),
$$

which is the Lyapunov condition for $S_{N}$.

$\operatorname{Re}$ (iii). Since $R_{2 r}$ are constant,

$$
\operatorname{var}\left(R_{N}\right)=\sum_{r} \operatorname{var}\left(R_{1 r}\right) / N^{2} \leqslant \sum_{r} E\left(R_{1 r}^{2}\right) / N^{2} \leqslant \epsilon^{2} /\left(N n^{2}\right) .
$$

This implies (iii), since $\epsilon$ can be made arbitrarily small. Q.E.D.

Remark. The asymptotic normality may be proved by applying the central limit theorem directly to $J_{1}$ in the form (1.1), not indirectly to $S_{N}$. This approach, however, requires asymptotic expansions of $E\left\{f^{2}\left(x_{r}\right)\right\}$ and $I_{r}$ up to the terms of order $n^{-2}$ so that a stronger assumption $f \in C^{2}$ is needed instead of $f \in C^{1}$. This remark is valid also with Theorems 2,3 and 4 for which a much stronger assumption $f \in C^{k}$ ( $k=4,4$ and 6 , respectively) is required.

THEOREM 2. Assume $f \in C^{2}$. As $N \rightarrow \infty, J_{2}$ is asymptotically normally distributed with mean I and variance $\tau_{N}^{2}\left(J_{2}\right)$ defined in (1.9).

Proof. Similarly as (2.1)

$$
J_{2}-I=\frac{1}{2 N} \sum_{r=1}^{N}\left\{f\left(x_{r}\right)+f\left(x_{r}^{\prime}\right)-2 I_{r}\right\} .
$$

Expanding $f\left(x_{r}\right)$ and $f\left(x_{r}^{\prime}\right)$ in Taylor series around the point $c_{r}$, we find

$$
\begin{aligned}
& f\left(x_{r}\right)=f_{r}+\sum_{i=1}^{s} \delta_{r}^{i} f_{r}^{i}+\frac{1}{2} \sum_{i, j=1}^{s} \delta_{r}^{i} \delta_{r}^{j} f_{r}^{i j}+R_{1 r} \\
& f\left(x_{r}^{\prime}\right)=f_{r}-\sum_{i=1}^{s} \delta_{r}^{i} f_{r}^{i}+\frac{1}{2} \sum_{i, j=1}^{s} \delta_{r}^{i} \delta_{r}^{j} f_{r}^{i j}+R_{1 r}^{\prime},
\end{aligned}
$$

where $\left|R_{1 r}\right|$ and $\left|R_{1 r}^{\prime}\right|$ are bounded from above by $\epsilon\left\|\delta_{r}\right\|^{2}$ for every $r$, provided $N$ is sufficiently large.

Taking one more term in the expansion (2.3), we have

$$
I_{r}=f_{r}+\sum_{i=1}^{s} \frac{1}{24 n_{i}^{2}} f_{r}^{i i}+R_{2 r}
$$

where $\left|R_{2 r}\right| \leqslant \epsilon / n^{2}$ for every $r$. Substitution of (2.9) and (2.10) into (2.8) yields 
where

$$
J_{2}-I=S_{N}+R_{N}
$$

$$
S_{N}=\frac{1}{2 N} \sum_{r=1}^{N}\left(\sum_{i, j=1}^{s} \delta_{r}^{i} \delta_{r}^{j} f_{r}^{i j}-\sum_{i=1}^{s} \frac{1}{12 n_{i}^{2}} f_{r}^{i i}\right)
$$

$$
R_{N}=\frac{1}{2 N} \sum_{r=1}^{N}\left(R_{1 r}+R_{1 r}^{\prime}-2 R_{2 r}\right)
$$

Just as for Theorem 1 we have only to prove the following three propositions:

(i) $\operatorname{var}\left(S_{N}\right) \sim \tau_{N}^{2}\left(J_{2}\right)$ as $N \rightarrow \infty$,

(ii) $S_{N}$ satisfies the Lyapunov condition, and

(iii) $\operatorname{var}\left(R_{N}\right)=o\left(\left(N n^{4}\right)^{-1}\right)$ as $N \rightarrow \infty$.

Part (i) is equivalent to the main theorem in Haber [2] or (1.8), while (ii) and (iii) can be verified by using reasoning similar to that in the proof of Theorem 1 . Q.E.D.

Corollary 1. For $k=1$ or 2 , assume $f \in C^{k}$. As $N \rightarrow \infty, J_{k}^{\prime}$ follows asymptotically a normal distribution with mean $I$ and variance

$$
\tau_{N}^{2}\left(J_{k}^{\prime}\right)=\frac{1}{2} \tau_{N}^{2}\left(J_{k}\right)
$$

3. Asymptotic Normality of $D_{1}$ and $D_{2}$. Though the asymptotic normality of $D_{1}$ or $D_{2}$ may not be so important in practice as that of any estimator of $I$ itself, it can be proved along a similar line of arguments for the latter. First let us consider $D_{1}^{2}$.

Theorem 3. Assume $f \in C^{1}$. As $N \rightarrow \infty, D_{1}^{2}$ is asymptotically normally distributed with mean $\operatorname{var}\left(J_{1}^{\prime}\right)$ and variance

$$
\tau_{N}^{2}\left(D_{1}^{2}\right)=\frac{1}{2880 N^{3}}\left\{7 \sum_{i=1}^{s} \frac{1}{n_{i}^{4}} \int_{G_{s}}\left(\frac{\partial f}{\partial x^{i}}\right)^{4}+10 \sum_{i \neq j=1}^{s} \frac{1}{\left(n_{i} n_{j}\right)^{2}} \int_{G_{s}}\left(\frac{\partial f}{\partial x^{i}} \frac{\partial f}{\partial x^{j}}\right)^{2}\right\}
$$

Proof. Similarly to (2.2) it is the case that

where

$$
f\left(z_{r}\right)=f_{r}+\sum_{i=1}^{s} \zeta_{r}^{i} f_{r}^{i}+R_{2 r}
$$

$$
\zeta_{r}=\left(\zeta_{r}^{1}, \ldots, \zeta_{r}^{S}\right)=z_{r}-c_{r}, \quad\left|R_{2 r}\right| \leqslant \epsilon\left\|\zeta_{r}\right\| \quad \text { for every } r
$$

Substitution of (2.2) and (3.1) into (1.5) yields

$$
D_{1}^{2}=T_{N}+R_{N}
$$

where

$$
\begin{aligned}
& T_{N}=\frac{1}{4 N^{2}} \sum_{r=1}^{N}\left(\sum_{i=1}^{s} \eta_{r}^{i} f_{r}^{i}\right)^{2}, \\
& R_{N}=\frac{1}{4 N^{2}} \sum_{r=1}^{N}\left\{\left(R_{1 r}-R_{2 r}\right)^{2}+2\left(R_{1 r}-R_{2 r}\right) \sum_{i=1}^{s} \eta_{r}^{i} f_{r}^{i}\right\}
\end{aligned}
$$

and $\eta_{r}^{i}=\delta_{r}^{i}-\zeta_{r}^{i}$. 
Since $E\left(D_{1}^{2}\right)=\operatorname{var}\left(J_{1}^{\prime}\right)$, a proof of Theorem 3 can be reduced to the following:

(i) $\operatorname{var}\left(T_{N}\right) \sim \tau_{N}^{2}\left(D_{1}^{2}\right)$ as $N \rightarrow \infty$,

(ii) $T_{N}$ satisfies the Lyapunov condition, and

(iii) $\operatorname{var}\left(R_{N}\right)=o\left(\left(N^{3} n^{4}\right)^{-1}\right)$ as $N \rightarrow \infty$.

$\operatorname{Re}(i)$. First we have

$$
\operatorname{var}\left(T_{N}\right)=\frac{1}{16 N^{4}} \sum_{r} \operatorname{var}\left(\sum_{i} \eta_{r}^{i} f_{r}^{i}\right)^{2}
$$

Since for every $i, j$ and $r$

$$
\begin{aligned}
E\left(\eta_{r}^{i}\right)^{2} & =\frac{1}{6 n_{\mathbf{i}}^{2}}, \quad E\left(\eta_{r}^{i}\right)^{4}=\frac{1}{15 n_{i}^{4}}, \\
E\left(\eta_{r}^{i} \eta_{r}^{j}\right)^{2} & =\frac{1}{36 n_{i}^{2} n_{j}^{2}} \quad(i \neq j) ;
\end{aligned}
$$

and since the expectations of any other monomial of the $\eta$ 's of order 2 or 4 vanish, we find

$$
\begin{aligned}
& E\left(\sum_{i} \eta_{r}^{i} f_{r}^{i}\right)^{2}=\frac{1}{6} \sum_{i}\left(\frac{f_{r}^{i}}{n_{i}}\right)^{2}, \\
& E\left(\sum_{i} \eta_{r}^{i} f_{r}^{i}\right)^{4}=\frac{1}{15} \sum_{i}\left(\frac{f_{r}^{i}}{n_{i}}\right)^{4}+\frac{1}{12} \sum_{i \neq j}\left(\frac{f_{r}^{i} f_{r}^{j}}{n_{i} n_{j}}\right)^{2} .
\end{aligned}
$$

This implies

$$
\operatorname{var}\left(\sum_{i} \eta_{r}^{i} f_{r}^{i}\right)^{2}=\frac{1}{180}\left\{7 \sum_{i}\left(\frac{f_{r}^{i}}{n_{i}}\right)^{4}+10 \sum_{i \neq j}\left(\frac{f_{r}^{i} f_{r}^{j}}{n_{i} n_{j}}\right)^{2}\right\} .
$$

Substitution of the last formula into (3.4) proves (i).

Re (ii). Define

$$
X_{N r}=\left(\sum_{i=1}^{s} \eta_{r}^{i} f_{r}^{i}\right)^{2}
$$

Then a straightforward calculation gives

$$
\sum_{r} E\left|X_{N r}\right|^{3}=O\left(N n^{-6}\right)
$$

and hence,

$$
\left[\sum_{r} E\left|X_{N r}\right|^{3}\right] /\left[\sum_{r} E\left(X_{N r}^{2}\right)\right]^{3 / 2}=O\left(N^{-1 / 2}\right)=o(1) .
$$

$\operatorname{Re}$ (iii). From (3.3) it follows that

$$
\left|R_{N}\right| \leqslant \frac{\epsilon^{2}}{4 N^{2}} \sum_{r}\left\{\left(\left\|\delta_{r}\right\|+\left\|\zeta_{r}\right\|\right)^{2}+2\left(\left\|\delta_{r}\right\|+\left\|\zeta_{r}\right\|\right) \frac{1}{n} \sum_{i}\left|f_{r}^{i}\right|\right\}
$$

and hence,

$$
\operatorname{var}\left(R_{N}\right) \leqslant E\left(R_{N}^{2}\right)=o\left(\left(N^{3} n^{2}\right)^{-1}\right) \text {. Q.E.D. }
$$

Remark. Though $\tau_{N}^{2}\left(J_{1}^{\prime}\right)$ defined in (2.12) is indeed the leading term in the asymptotic expansion of $\operatorname{var}\left(J_{1}^{\prime}\right)$ in $n^{-1}$, the phrase "mean $\operatorname{var}\left(J_{1}^{\prime}\right)$ " in the statement 
of Theorem 3 cannot be replaced by "mean $\tau_{N}^{2}\left(J_{1}^{\prime}\right)$ ", in general. The reason is that the difference $\operatorname{var}\left(J_{1}^{\prime}\right)-\tau_{N}^{2}\left(J_{1}^{\prime}\right)$ is $o\left(\left(N n^{2}\right)^{-1}\right)$ and hence is not negligible in general as compared with $\tau_{N}\left(D_{1}^{2}\right)$ which is of order $\left(N^{3 / 2} n^{2}\right)^{-1}$.

Similarly, we can prove the following:

Theorem 4. Assume $f \in C^{2}$. As $N \rightarrow \infty, D_{2}^{2}$ is asymptotically normally distributed with mean $\operatorname{var}\left(J_{2}^{\prime}\right)$ and variance $\tau_{N}^{2}\left(D_{2}^{2}\right)$ which is a linear combination of seven terms of the form

$$
\sum_{i, j, k, l=1}^{s} \frac{1}{N^{3}\left(n_{i} n_{j} n_{k} n_{l}\right)^{2}} \int_{G_{s}}\left(\frac{\partial^{2} f}{\partial x^{i} \partial x^{j}} \frac{\partial^{2} f}{\partial x^{k} \partial x^{l}}\right)^{2},
$$

where some of $i, j, k$ and $l$ are constrained to be equal.

The root-square transformation of the random variable $D_{k}^{2}(k=1,2)$ induces the following:

Corollary 2. For $k=1$ or 2 , assume $f \in C^{k}$. As $N \rightarrow \infty, D_{k}$ is asymptotically normally distributed with mean $\sigma\left(J_{k}^{\prime}\right)$ and variance $1 / 4 \tau_{N}^{2}\left(D_{k}^{2}\right) / \operatorname{var}\left(J_{k}^{\prime}\right)$, where $\sigma\left(J_{k}^{\prime}\right)$ stands for the standard deviation of $J_{k}^{\prime}$.

Department of Applied Mathematics

Faculty of Engineering Science

Osaka University

Toyonaka, Osaka 560, Japan

1. S. HABER, “A modified Monte-Carlo quadrature," Math. Comp., v. 20, 1966, pp. 361368. MR $35 \# 1178$.

2. S. HABER, “A modified Monte-Carlo quadrature. II," Math. Comp., v. 21, 1967, pp. 388-397. MR 38 \# 2922.

3. J. M. HAMMERSLEY \& K. W. MORTON, "A new Monte Carlo technique: Antithetic variates," Proc. Cambridge Philos. Soc., v. 52, 1956, pp. 449-475. MR 18, 336.

4. T. KITAGAWA, "Random integrations," Bull. Math. Statist., v. 4, 1950, pp. 15-21. MR 14, 457.

5. M. LOEVE, Probability Theory, 3rd ed., Van Nostrand, Princeton, N.J., 1963. MR 34 \# 3596. 